\title{
Double bundle medial patellofemoral ligament reconstruction
}

Volume 9 Issue 5 - 2017

\author{
David Sadigursky \\ Manoel Victorino Hospital, Faculty of the COT Hospital, Brazil
}

Correspondence: David Sadigursky, Manoel Victorino Hospital/Faculty of the COT Hospital, Salvador, Bahia, Brazil, Email davidsad@gmail.com

Received: October 13, 2017 | Published: December 22, 2017
The medial patellofemoral ligament (MPFL) is the major restraint that prevents lateral translocation of the patella. In most patients, the MPFL is torn during acute patellar dislocation. Because of this, several surgical techniques have been described for the treatment of patellar instability. Surgical treatment is accepted as necessary for the restoration of the stability of the MPFL. ${ }^{1}$

Despite the biomechanical importance of MPFL reconstruction, its value was appreciated only relatively recently, particularly over the last two decades. ${ }^{2}$ Although a wide range of techniques have been described for MPFL reconstruction, with different graft sources and fixation methods, accumulating evidence shows good clinical results of this surgery, with a very low recurrence rate of instability. ${ }^{1,3,4}$

A major concern is the consequence of a graft positioning error in MPFL reconstruction. Small errors of $5 \mathrm{~mm}$ in the ideal position or graft tension $>2 \mathrm{~N}$ are known to lead to increased joint forces on the medial facet of the patella, increasing the risk of pain and degeneration of the patellar cartilage. This leads to the need for techniques that can distribute the graft tension on the patella, reproducing the anatomy more closely. ${ }^{5}$

However, Sandmeier et al. ${ }^{4}$ and Parker et al. ${ }^{6}$ showed that isolated MPFL reconstruction was unable to restore normal patellar tracking through medium and maximum flexion. Failure to restore proper anatomy or isometry of the MPFL may be responsible for this issue, thus limiting the long-term success of such reconstruction.

In 2010, Kang et al. ${ }^{7}$ introduced the concept of MPFL bands. The ligament has a thin layer that connects the femoral condyle to the supramedial border of the patella. From the femoral origin, MPFL fibers become larger, forming two bands, named by the authors as the inferior straight bundle (ISB) and superior oblique bundle (SOB). However, the two bands do not separate completely, forming a single structure. The lower fibers act as the static restraint, and the upper fibers act in the dynamic stabilization of the patella owing to their close association with the vastus medialis obliquus tendon.

MPFL reconstruction has become the technique of choice for most authors since the 1990s, even in cases where trochlear dysplasia and patella alta are identified. Isolated MPFL reconstruction has been shown to be a suitable and effective technique for the correction of patellar instability, except in cases where the tibial tuberositytrochlear groove (TTTG) distance is longer than $20 \mathrm{~mm}$.

In 2010, Kang et al. ${ }^{7}$ described the anatomy and function of the MPFL and their two bands. The superior oblique bundle together with the vastus medialis obliquus exerts traction on the patella, medially promoting a dynamic restraint. Meanwhile, the lower inferior straight bundle acts as a static restraint to balance the resulting lateral forces that act on the patella, caused by the Q angle. Thus, reconstruction of the two bands could increase the stability during the first flexion angles around $30^{\circ}$. The function of the MPFL at greater angles requires more elucidative studies. ${ }^{8-15}$

Despite the MPFL being the primary stabilizer against patellar dislocation between $30-40^{\circ}$ of flexion, Philippot et al. ${ }^{16}$ described the role of the medial patellotibial (MPTL) and the medial patellomeniscal (MPML) ligaments acting on the rotation, tilt, and dislocation at angles greater than $45^{\circ}$. The reconstruction of the MPTL is gaining popularity both in patients with open physes and in adults $(16,17)$. During the arc of motion from total extension to flexion of $90^{\circ}$, the MPTL and MPML contribute $28 \%-48 \%$ against the lateralization of the patella, $23 \%-71 \%$ against tilt, and $32 \%-92 \%$ against rotation. This finding cannot be disregarded in the planning of the correction of patellar instability. ${ }^{16,17}$ Despite the growing interest in the reconstruction of MPTL, with the aim of reducing dislocation of the patella at larger angles, possibly eliminating the inverted J sign, no data so far show the superiority of clinical outcomes in the combined reconstruction of the MPFL and MPTL with the double- or single-bundle technique.

Applying the MPFL reconstruction technique with the use of metal anchors has shown that the resistance is appropriate for achieving restraint against dislocation of the patella, especially in the early knee flexion angles. The MPFL tensile strength is relatively low, around $208 \mathrm{~N},{ }^{2}$ making the implant useful in fixing the graft. Mountney et al. ${ }^{18}$ showed no significant difference in tensile strength between the passage of the graft through bone tunnels and anchor fixation. Comparing the bone tunnels and anchor fixation, Hapa et al. ${ }^{19} \mathrm{did}$ not find differences, although anchor fixation showed less stiffness. Bone tunnels were also demonstrated to enable better integration of the graft. As stated by Song et al. ${ }^{9}$ tendon-to-bone healing also occurs in the anchor fixation technique, as a bone bed in the medial border of the patella is made to accommodate the graft, which is then covered by the layer of periosteum and medial retinaculum.

Patellar fracture and cartilage perforation were described as among the possible complications of the patellar bone tunnel fixation technique. These complications were not found in studies that used anchor fixation. This fact is due to the non-perforation of the patella 
with drills, which require greater technical precautions in order to avoid these complications, particularly in patients with smaller patellae, such as Asians or people of short stature..$^{20-21}$

An advantage of using the anchor fixation technique is the possibility of using a graft of shorter length, as in the case of the gracilis tendon. Furthermore, the procedure allows for smaller incisions with better aesthetic results. This is because less exposure is required, as looping the graft through the lateral facet of the patella or to expose the bone tunnels is not necessary. Corroborating the opinion of Song et al. ${ }^{9}$ another advantage is that it is a relatively simple technique, which results in shorter surgical time and decreased exposure to radiation.

Straight lateral radioscopy was used to identify the femoral insertion site of the graft in all cases, following the parameters described by Schöttle et al..$^{14}$ According to studies by Schöttle et al. ${ }^{22}$ the use of radioscopy is considered of great importance in determining the ideal anatomical point for graft fixation in the femur. Several authors have shown that non-anatomical positioning of the femoral tunnel interferes with the patellofemoral kinematics. Elias \& Cosgarea $^{5}$ found that the tunnel positioning error, more proximal in the femur, can overload the medial compartment of the patella, which can lead to degeneration of the medial facet and graft rupture or failure. Similarly, Thaunat \& Erasmus $^{23}$ suggested that non-proximal anatomical positioning of the graft would lead to hypertensioning of the graft and consequently to knee rigidity. A more distal positioning is not able to tension the graft enough to restrict dislocation of the patella. Amis et al. ${ }^{2}$ found that the positioning error in the frontal plane is better tolerated.

Kang et al. ${ }^{7}$ suggested that the fixation of the MPFL bands should be performed at different angles, with the ISB at $0^{\circ}$ and the SOB at $30^{\circ} .{ }^{24}$ In this way, the patellar stability can be maintained at higher flexion angles such as at greater than $60^{\circ}$.

\section{Surgical technique}

The patient was placed in the supine position under spinal anesthesia. First arthroscopy was performed to identify associated lesions or to remove intra-articular loose bodies. Lateral retinacular release was not performed in any of the cases.

The gracilis tendon was removed through an incision of about 2 to $3 \mathrm{~cm}$ above the pes anserine tendons. The "goose foot" bursa was removed, and the semitendinosus tendon was exposed and resected. The gracilis tendon was considered sufficient due to its resistance, which is compatible with what is expected for MPFL, which is $208 \mathrm{~N}$. However, the semitendinous tendon was used, as it was long enough to permit the preparation of the two bundles, with the passage of the graft through the bone tunnel in the femur.

Then, an incision was made under the medial border of the patella at a distance of 4 to $5 \mathrm{~cm}$ between the medial border of the patella and the medial epicondyle of the femur. With the aid of a curette, a sulcus was made on the medial border of the patella, above the transition with the posterior chondral facet, deep enough to accommodate the graft. Two 5-mm metal anchors were inserted into the proximal twothirds of the patella, 10 to $15 \mathrm{~mm}$ from the joint.

The anatomical site on the femur was confirmed with the aid of straight lateral radioscopy, following the parameters described by Schöttle et al. ${ }^{14} \mathrm{~A}$ Kirschner wire was inserted at this point, directed anteriorly and proximally, thus avoiding penetration into the posterior region of the femoral condyle. The femur was drilled to create the bone tunnel, and the central part of the graft was first fixed at this point by using the bioabsorbable interference screw. Next, the two bundles of the graft were passed through the patella by the second layer of the medial retinaculum, with the aid of curved forceps. The two ends of the graft were sutured separately by the anchors. The first bundle, considered as the inferior straight bundle, was set at $30^{\circ}$ of flexion; and subsequently, the superior oblique bundle was set at $60^{\circ}$ of flexion. ${ }^{7,15,24}$ The required tension was checked by the mobility of the patella, which can reach glide approximately 2 quadrants.

After fixation, the position of the patella was checked by using arthroscopic imaging. Local irrigation was performed with $0.9 \%$ physiological saline, the subcutaneous tissue was sutured with Vicryl 2.0, and the skin was sutured with separate stitches of Nylon 3.0 [Figures 1-3].

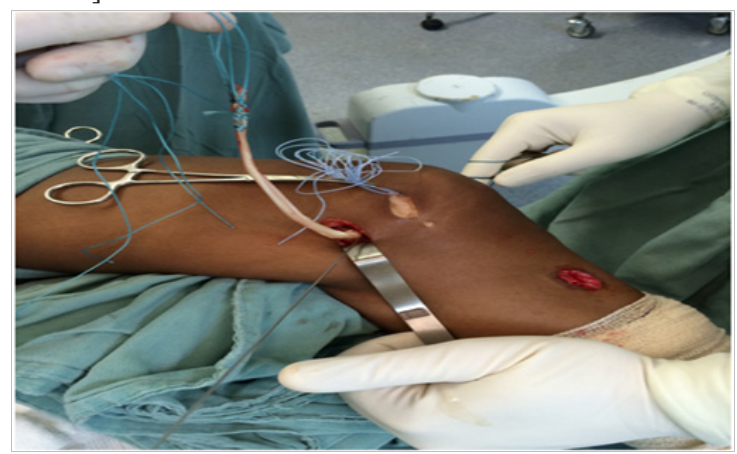

Figure I Image showing the graft fixation at the anatomical point in the femur first.

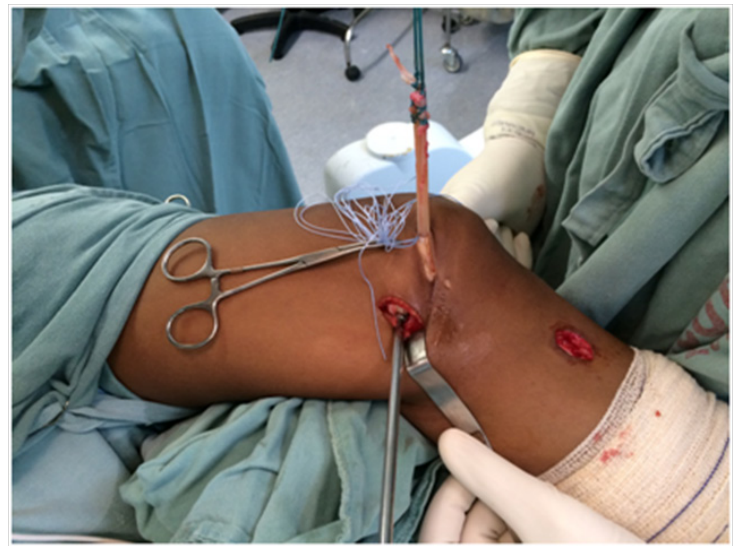

Figure 2 Image showing the fixation of the graft in the femoral tunnel with metallic interference screw.

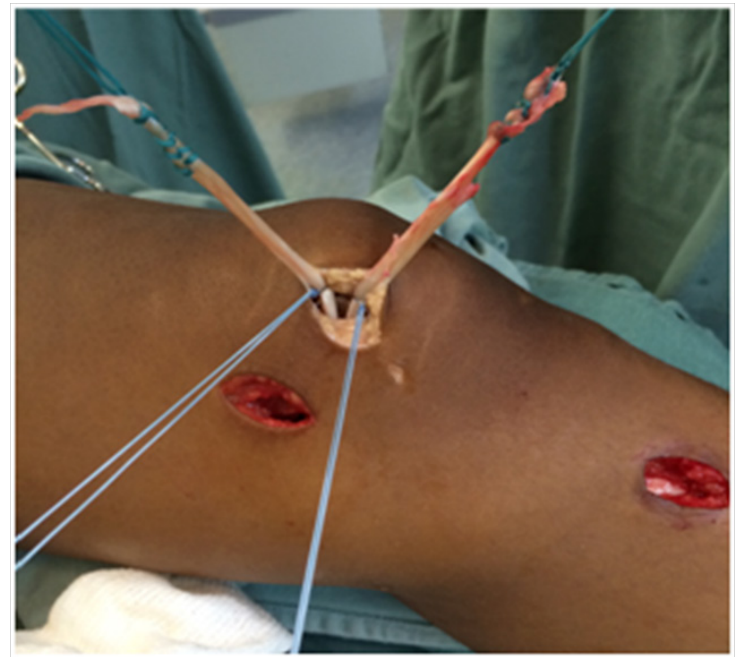

Figure 3 Image demonstrating the fixation of the inferior transverse band fixation followed by the upper oblique band at independent knee flexion angles, 30 and 60 degrees respectively. 


\section{Postoperative period}

The knee was immobilized in extension with a long brace for 2 weeks. Physical therapy was initiated in the first week with a progressive increase in the arc of motion and should reach $120^{\circ}$ of flexion during the sixth week. During the first 3 weeks, partial load with the aid of crutches was allowed. After the third week, the patient was allowed to bear full load without crutches. Isometric exercises to strengthen the quadriceps and elevation of the leg in extension were permitted in the immediate postoperative period. After 6 weeks, the patient should be able to return to normal activity. Contact sports and rotation were allowed at 6 months after surgery.

\section{Conclusion}

The double bundle MPFL reconstruction at different angles of flexion, or at the same angle fixing on the patella first, aims to gain stability at higher flexion angles, especially above 45 degrees of knee flexion. Nevertheless more clinical trials should be conducted in the future, eszpecially with the combined medial patellotibial ligament reconstruction, which seems to have similar goals in adult patients. In addition, more precise stratification of the patients should be considered for the elective patients that should undergo the technique.

\section{Acknowledgements}

None.

\section{Conflicts of interest}

None.

\section{References}

1. Colvin AC, West RV. Patellar instability. J Bone Joint Surg Am. 2008;90(12):2751-2762.

2. Amis AA, Firer P, Mountney J, et al. Anatomy and biomechanics of the medial patellofemoral ligament. Knee. 2003;10(3):215-220.

3. Schottle PB, Hensler D, Imhoff AB. Anatomical double-bundle MPFL reconstruction with an aperture fixation. Knee Surg Sports Traumatol Arthrosc. 2010;18(2):147-151.

4. Sandmeier RH, Burks RT, Bachus KN, et al. The effect of reconstruction of the medial patellofemoral ligament on patellar tracking. Am J Sports Med. 2000;28(3):345-349.

5. Elias JJ, Cosgarea AJ. Technical errors during medial patellofemoral ligament reconstruction could overload medial patellofemoral cartilage: a computational analysis. The American journal of sports medicine. 2006;34(9):1478-1485.

6. Parker DA, Alexander JW, Conditt MA, et al. Comparison of isometric and anatomic reconstruction of the medial patellofemoral ligament: a cadaveric study. Orthopedics. 2008;31(4):339-343.

7. Kang HJ, Wang F, Chen BC, et al. Functional bundles of the medial patellofemoral ligament. Knee Surg Sports Traumatol Arthrosc. 2010;18(11):1511-1516.

8. Lenschow S, Schliemann B, Gestring J, et al. Medial patellofemoral ligament reconstruction: fixation strength of 5 different techniques for graft fixation at the patella. Arthroscopy. 2013;29(4):766-773.
9. Song SY, Kim IS, Chang HG, et al. Anatomic medial patellofemoral ligament reconstruction using patellar suture anchor fixation for recurrent patellar instability. Knee Surg Sports Traumatol Arthrosc. 2013;22(10):2431-2437

10. Phillips CL, Silver DA, Schranz PJ, et al. The measurement of patellar height: a review of the methods of imaging. J Bone Joint Surg Br. 2010;92(8):1045-1053.

11. Dejour H, Walch G, Nove-Josserand L, et al. Factors of patellar instability: an anatomic radiographic study. Knee Surg Sports Traumatol Arthrosc. 1994;2(1):19-26.

12. Kujala UM, Osterman K, Kormano M, et al. Patellofemoral relationships in recurrent patellar dislocation. The Journal of bone \& joint journal. 1989;71(5):788-792.

13. Smith TO, Davies L, O'Driscoll ML, et al. An evaluation of the clinical tests and outcome measures used to assess patellar instability. Knee. 2008;15(4):255-262.

14. Schottle PB, Schmeling A, Rosenstiel N, et al. Radiographic landmarks for femoral tunnel placement in medial patellofemoral ligament reconstruction. Am J Sports Med. 2007;35(5):801-804.

15. Sadigursky D GR, Pereira CAM, Pécora JR, et al. Método de Ensaio Biomecânico para Análise da Isometricidade na Reconstrução do Ligamento Patelofemoral Medial. Rev Bras Ortop. 2012;47598-605(5):598-605.

16. Philippot R, Boyer B, Testa R, et al. The role of the medial ligamentous structures on patellar tracking during knee flexion. Knee surgery, sports traumatology, arthroscopy: official journal of the ESSKA. 2012;20(2):331-336.

17. Sobhy MH, Mahran MA, Kamel EM. Midterm results of combined patellofemoral and patellotibial ligaments reconstruction in recurrent patellar dislocation. European journal of orthopaedic surgery \& traumatology: orthopedie traumatologie. 2013;23(4):465-70.

18. Mountney J, Senavongse W, Amis AA, et al. Tensile strength of the medial patellofemoral ligament before and after repair or reconstruction. The Journal of bone \& joint surgery. 2005;87(1):36-40.

19. Hapa O, Aksahin E, Ozden R, et al. Aperture fixation instead of transverse tunnels at the patella for medial patellofemoral ligament reconstruction. Knee surgery, sports traumatology, arthroscopy. 2012;20(2):322-326.

20. Arendt EA, Dejour D. Patella instability: building bridges across the ocean a historic review. Knee surgery, sports traumatology, and arthroscopy. 2013;21(2):279-293.

21. Steiner TM, Torga-Spak R, Teitge RA. Medial patellofemoral ligament reconstruction in patients with lateral patellar instability and trochlear dysplasia. Am J sports med. 2006;34(8):1254-1261.

22. Servien E, Fritsch B, Lustig S, et al. In vivo positioning analysis of medial patellofemoral ligament reconstruction. Am J sports med. 2011;39(1):134-139.

23. Thaunat M, Erasmus PJ. Management of overtight medial patellofemoral ligament reconstruction. Knee Surg Sports Traumatol Arthrosc. 2009; 17(5):480-483.

24. Han H, Xia Y, Yun X, et al. Anatomical transverse patella double tunnel reconstruction of medial patellofemoral ligament with a hamstring tendon autograft for recurrent patellar dislocation. Arch Orthop Trauma Surg. 2011;131(3):343-351. 